\title{
Side effects of triazoles on treated crops
}

\author{
Michal Jakl ${ }^{1}$, Sanja Ćavar Zeljković ${ }^{2}$ Ishak Kovač ${ }^{3}$, Kateřina Bělonožníková ${ }^{4}$, and Jana \\ Jaklová Dytrtováa ${ }^{3}$ \\ ${ }^{1}$ Czech University of Life Sciences Prague Faculty of Agrobiology Food and Natural \\ Resources \\ ${ }^{2}$ Palacky University Olomouc Faculty of Science \\ ${ }^{3}$ Institute of Organic Chemistry and Biochemistry Czech Academy of Sciences \\ ${ }^{4}$ Charles University Faculty of Science
}

March 18, 2021

\begin{abstract}
Triazolic fungicides are widely applied in crop production to protect plants against fungal pathogens. However, they may influence the biochemical processes in plants and other non-target species. This paper is aimed at the effect of triazoles (namely tebuconazole, cyproconazole, and penconazole) single/mixed applications on the phenolics production in tomato (Solanum lycopersicum L.) fruit peel, amount of chlorophyll $a$ and $b$ in tomato leaves as well as on basic plant growth parameters. For this purpose, cherry tomatoes were planted in the pot experiment and foliarly-treated weekly, with the same total triazoles dose of $3.52 \mu \mathrm{mol}$ per plant (in mixtures of 1.71 or $1.17 \mu \mathrm{mol}$ of each in two or three components, respectively). The treatments increased the weight of fruits in the $1^{\text {st }}$ harvest about $43 \%$, however, this effect was not observed in the next harvest. Increased oxidative stress in the triazoles presence was observed, based on the elevated production of antioxidant phenolics in the $1^{\text {st }}$ harvest. Most alarming is the decrease of the weight of thin stems and foliage and the concentration of chlorophyll $a(b)$ in leaves in all triazoles-treated variants. The non-target impacts on plant biochemical processes (related to the phenolics or chlorophylls production and functionality) were confirmed.
\end{abstract}

\section{Keywords}

chlorophylls; foliar treatment; group interactions; phenolic compounds; photosynthesis; redox conditions; Solanum lycopersicum ; tomato; triazolic mixtures

\section{Acknowledgement}

This work was finantialy supported by the Czech Science Foundation project number 18-01710S (J.J.D.).

\section{Graphical abstract}




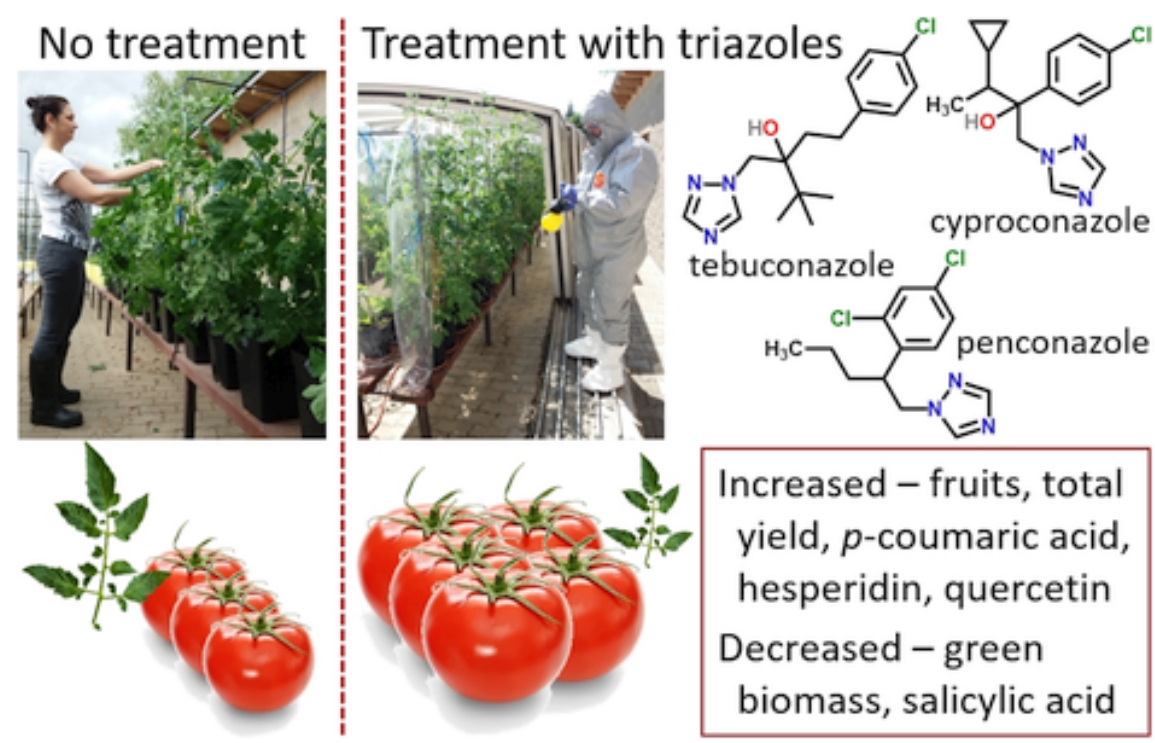

